\title{
Modeling of the Technological Process for Metal Production using a Probability Finite Automatic Machine
}

\author{
Korneev A.M., Sukhanov A.V., Abdullah L.S., Shipulin I.A.
}

\begin{abstract}
To date, the popularity of areas such as system management and the development of automated control systems using finite state machines has grown significantly, while the degree of mutual integration of these areas is quite high. So, in each of them the creation of decision-making systems and software systems of intellectual support is required. And the most frequently used tool for this in modern research and scientific projects is state machines - finite discrete or probabilistic automata, although they often do not allow the creation of decision-making systems quite efficient without large labor costs. The subject of this study is to create a probabilistic finite state machine capable of accurately predicting the properties of alloys, and thereby provide intelligent decision support in the process of manufacturing metal products.
\end{abstract}

Keywords: finite state machine, probabilistic automaton, discrete automaton, transition matrix, technological process, technological circuit cell.

\section{INTRODUCTION}

Systems with an invariable discrete clock cycle and characterized by discrete information processing are classified as multi-cycle systems with a discrete input. The computer implementation of multi-cycle systems differs significantly from the implementation of conventional systems, since the former have a specific representation structure, schematization, and mathematical apparatus for description $[1,2]$. In accordance with the theory of systems, discrete systems are divided into the following subsystems: organizational, managerial and executive. The first subsystem selects the target and the corresponding optimality criteria. The second subsystem is also sometimes called informational, since it is responsible for describing the interaction of a discrete system with the external environment, as well as for the interaction of individual elements of the system with each other. The third subsystem consists of functionals responsible for performing the operations necessary to achieve the goal chosen by the first, organizational subsystem [1].

The basic concept of the theory of discrete systems is the cell interpretation, on which the concept of linear cellular machines is based. A lot of discrete-argument models studied so far can be represented in the form of cell configurations of various structures composed of discrete blocks of the following types: discrete-time dynamic system (cell with memory), combinational circuit (cell without memory) [3].

Revised Manuscript Received on November 05, 2019.

Korneev A.M., Lipetsk State Technical University Sukhanov A.V., Lipetsk State Technical University Abdullah L.S., Lipetsk State Technical University Shipulin I.A. Lipetsk State Technical University
In [4], it is proposed to understand a separate distribution block as a discrete system for which an algorithmic functional (or analytical operator) is constructed Algorithmic functionality compares the output signal for each input signal, so the described method of discreteargument models is convenient to use when modeling multistage technological processes. Each individual cell in this case will correspond to any aggregate. By presenting technological parameters of metal products (chemical composition, heat treatment parameters, cost of raw materials costs) as a set of hotel cells with many inputs, states, and outputs, it is possible to construct mathematical, simulation, and computer models of multi-stage production processes $[5,6,7]$.

Thus, the control of technological processes in the production of metal products can be carried out on the basis of a discrete argument approach using probabilistic automata and iterative circuits, which make it possible to represent the system in the form of a finite automaton, whose internal state changes in a given framework [8].

\section{RESEARCH METHOD}

By probabilistic finite state machine (PFSM) is understood a discrete information converter, the functional transformations inside of which depend on the state of memory. The functioning of such a finite state machine can be described using probability functions [1, 9]. Unlike deterministic cellular automata, in which each pair of state and input values is uniquely associated with a pair of output and new state values, the probabilistic automaton sets only the probabilities of transition to a new state with known values of input and state.

We introduce the following notation. Let $V$ - the set of possible input signals $v[t]\left(v[t] \in V, t \in Z_{0}\right)$, Then $\delta_{\alpha}$ is a variant of a combination of input alphabets. Let $X$ be the set of possible states $x[t]\left(x[t] \in X, t \in Z_{0}\right)$, then $\zeta_{\beta}^{\prime}, \zeta_{\beta}$ are variants of combinations of state alphabets. Let $Y$ be the set of possible outputs $y[t]\left(y[t] \in Y, t \in Z_{0}\right)$, then $\tau_{\gamma}$ are variants of combinations of output alphabets. In these notations, the probabilistic automaton will be determined as follows. If the automaton is in the state $\zeta_{\beta}^{\prime}$, and the signal $\delta_{\alpha}$ arrives at its input, then it will transition to the state $\zeta_{\beta}$ with the output $\tau_{\gamma}$ with a probability equal to $P_{\beta, \gamma}^{<\beta^{\prime}, \alpha>}$ :

$$
P\left(\frac{\varsigma_{\beta}, \tau_{\gamma}}{\varsigma_{\beta}^{\prime}, \delta_{\alpha}}\right)=P_{\beta, \gamma}^{<\beta^{\prime}, \alpha>} .
$$

The initial state is specified in the form of probabilities with which any 
possible state can be the first [1]:

$$
P_{(0)}\left(\varsigma_{\beta}\right)=P_{\beta,(0)} \text {. }
$$

The probability distribution on the final alphabet of states must satisfy the conditions:

$$
0 \leq P_{\beta,(0)} \leq 1 ; \sum_{\varsigma_{,} \in X} P_{\beta,(0)}=1
$$

The functioning of the PFSM is based on the probability distribution given for each pair $\left(\zeta_{\beta}^{\prime}, \delta_{\alpha}\right)$ from the product of the sets $\mathrm{X}$ and $\mathrm{Y}$. The number of distributions coincides with the number of possible combinations $\left(\zeta_{\beta}^{\prime}, \delta_{\alpha}\right)$; at the same time, the following conditions must be satisfied:

$$
0 \leq P_{\beta, \alpha}^{<\beta, \alpha>} \leq 1 ; \sum_{\varsigma_{\mu} \in X} \sum_{\tau \in Y} P_{\beta, \alpha}^{<\beta, \alpha>}=1 .
$$

The laws of the distribution of outputs and states for a probabilistic finite state machine can be represented in the form of a table (table 1).

Table 1. Laws of distribution of states and outputs for PFSM

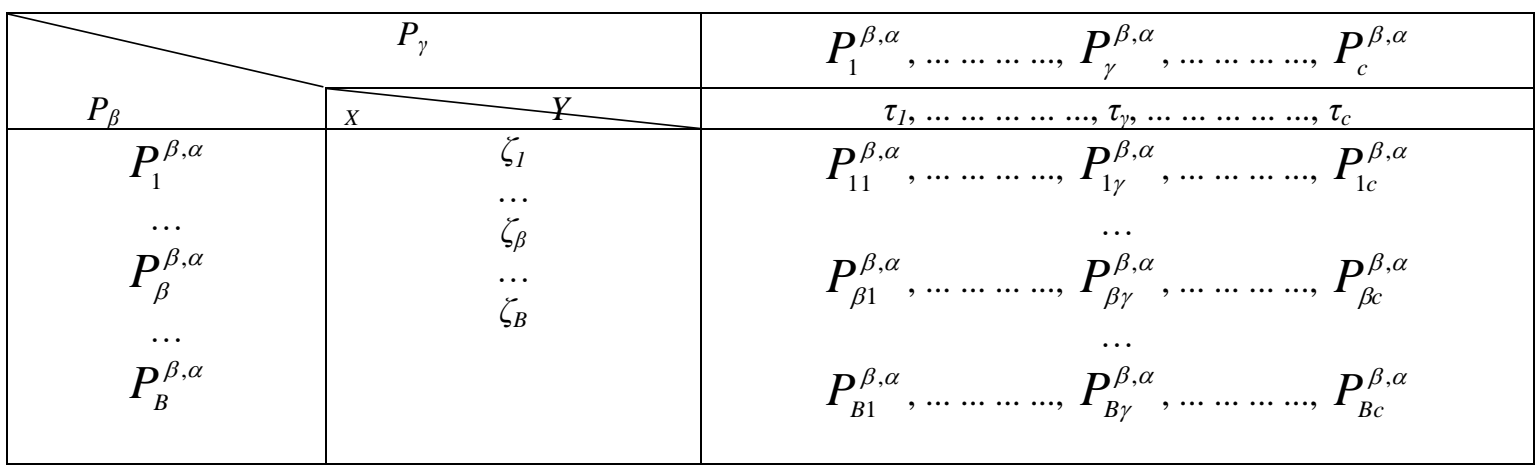

The states and outputs in the probabilistic finite state machine described using table 1 obey the particular distribution laws that determine the probabilities of the state $\zeta_{\beta}$ for the condition that the initial state was $\zeta_{\beta}^{\prime}$ and the signal $\delta_{\alpha}$ was input:

$$
\begin{gathered}
P_{\beta}^{<\beta^{\prime}, \alpha>}=\sum_{\tau, Y} P_{\beta, \gamma}^{<\beta^{\prime}, \alpha>} ; 0 \leq P_{\beta}^{<\beta^{\prime}, \alpha>} \leq 1 ; \\
\sum_{\mathcal{S}_{e} \in X} P_{\beta}^{<\beta^{\prime}, \alpha>}=1 .
\end{gathered}
$$

The relationships for determining similar output probabilities are:

$$
\begin{gathered}
P_{\gamma}^{<\beta \alpha>}=\sum_{\varsigma_{\mu} \in X} P_{\beta, \alpha}^{<\beta \alpha>} ; 0 \leq P_{\gamma}^{<\beta \alpha>} \leq 1 ; \\
\sum_{\tau, \in Y} P_{\gamma}^{<\beta \alpha>}=1 .
\end{gathered}
$$

The last relations allow us to determine the probability of the appearance of the output $\tau_{\gamma}$ under conditions when the initial state was $\zeta_{B}^{\prime}$, and the signal $\delta_{\alpha}$ was input.

A probabilistic automaton will be considered a Miles automaton when the following conditions are met:

$$
\begin{gathered}
P_{\beta, \gamma}^{<\beta^{\prime}, \alpha>}=P_{\beta}^{<\beta^{\prime}, \alpha>} * P_{\gamma}^{<\beta^{\prime}, \alpha>}, \delta_{\alpha} \in V, \tau_{\gamma} \in Y, \\
\varsigma_{\beta}^{\prime}, \varsigma_{\beta} \in X .
\end{gathered}
$$

In the probabilistic Miles automaton, the output signal and the new state are independent random variables with different distribution functions. In the probabilistic Moore automaton, the output signal is determined only depending on the current state and does not depend on the signal at the input [1]. For a probabilistic Moore automaton, the following condition must be fulfilled:

$$
P_{\beta, \gamma}^{<\beta^{\prime}, \alpha>}=P_{\beta}^{<\beta^{\prime}, \alpha>} * P_{\gamma}^{<\beta>} .
$$

In practice, a probabilistic automaton is most conveniently implemented in the form of a Markov chain, a random discrete process with the following properties:
1) The process $x[t]$ at any time $t \in Z_{0}$ takes a value from $X$ - a finite set of states $\left(X=\left\{\zeta_{B}\right\}\right)$.

\begin{tabular}{|c|c|}
\hline & $\zeta_{1}, \ldots \ldots \ldots \ldots \ldots \ldots \ldots \ldots \ldots \ldots \ldots \ldots \ldots \ldots \ldots \ldots \ldots \ldots \ldots \ldots \ldots \ldots \ldots \ldots \ldots, \zeta_{B}$ \\
\hline $\begin{array}{c}\zeta_{1}^{\prime} \\
\ldots \\
\ldots \\
\zeta_{\beta}^{\prime} \\
\ldots \\
\zeta_{B}^{\prime}\end{array}$ & 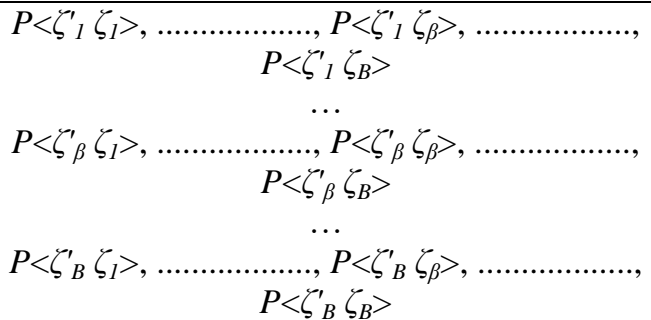 \\
\hline
\end{tabular}

2) At each moment of time, if at the previous moment there was a state $\zeta_{B}^{\prime}$, the probability of transition to the next state $\zeta_{B}$ depends only on the state $\zeta_{B}^{\prime}$.

The transition of a discrete finite Markov chain from a state at the previous moment of time (at time $t$-1) to a new state (at time $t$ ) is described using a stochastic transition matrix (table 2).

Table 2. Transition matrix for a discrete Markov finite chain

The transition matrix will be $X^{*} X$ in size and will not contain information on inputs and outputs [1]. Each row of the matrix, presented in the form of table 2, contains a set of probabilities of transitions from any state $\zeta_{\beta}$ to any subsequent state $\zeta_{\beta}^{\prime}$. It is obvious that the sum of the probability values of such transitions for each row is equal to one:

$$
\sum_{\varsigma \beta} P_{\varsigma \beta, \xi^{\prime} \beta}^{[t-1, t]}=1
$$

A probabilistic finite state machine in the form of a discrete Markov chain can be used to model technological processes in which random factors play an important role. Under conditions of uncertainty, when decisionmaking in the process control 
depends on a combination of many random factors, the stochastic transition matrix of the probabilistic automaton can serve as an adequate decision-making tool $[1,2,10]$.

\section{RESULTS AND ANALYSIS}

The work [11] describes the methodology for using iterative circuits for modeling multi-stage production systems. Here, iterative chains based on finite cellular automata were used to model systems. In this case, complex, multi-level processes were simulated, for which purpose the splitting of the general chain into separate cells, which are elementary automata, was used $[1,11]$.

The process itself is represented as flows. Elementary automata describe here separate functionals - aggregates and processing stages. A fully spatially distributed system synthesized using a linear chain of finite automata is described by the equations:

$$
\begin{aligned}
& x(k)=\Phi x(k-1)+\Psi v(k), \\
& y(k)=H x(k-1)+G v(k) .
\end{aligned}
$$

Modeling of the internal elementary cells of the chain, each of which is represented by a separate finite state machine, is presented in Figure 1.

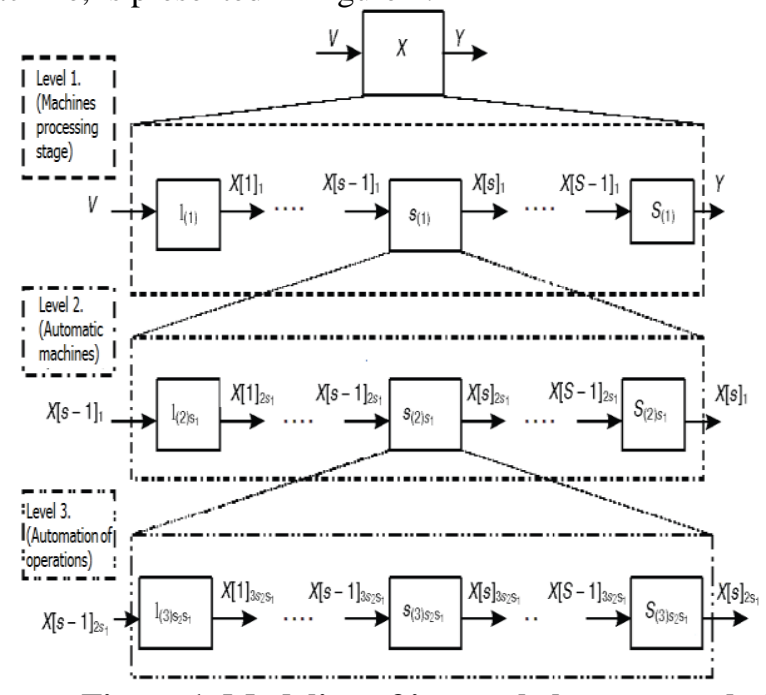

Figure 1. Modeling of internal elementary chain cells

Within each such elementary circuit, the process parameters are implemented sequentially. Each individual process is controlled on the basis of information obtained at the lower stage of the hierarchy, that is, for each unit cell of the chain (which corresponds to a separate stage of the process), technological quantities, including stochastic ones, are implemented sequentially $[1,11]$.

In the system, the interaction of elements always plays a significant role. At the same time, it is almost impossible to fully understand the essence of the system, if you do not consider the interaction of its elements with the environment.

The production of products that meet specified quality criteria or specified properties is not guaranteed under real conditions due to the influence of a significant number of random factors and parameters not taken into account. This means that in the case of representing the production process in the form of elementary finite state machines with a set of certain sets (alphabets) of input values and states, the inclusion of output quantities in the set of specified values of the alphabet of outputs is not guaranteed. Using probabilistic finite automata allows us to take into account a similar situation. For this, a separate study of the technological process is carried out to identify patterns in the distribution of technological factors, and then to determine the probabilities that specify the appearance of each possible state $\zeta \beta$ in production.

Alphabets containing the parameters of technological production determine the trajectory of the technological process, the sequence of operations that affect the change in the properties of the final product. Many of these parameters can be random in nature, therefore, to describe the influence of a certain parameter on the quality and properties of the product, probabilistic finite state machines can be used. The functioning of the PFSM is interpreted as follows: for each pair $\left(\zeta_{\beta}, \delta_{\alpha}\right)$, the probability distribution of states and outputs is specified $[1,11]$.

To optimize the structure and properties of metal products in a multi-stage technological process, we single out one level, but which it is necessary to decide on the chemical composition of the charge and the parameters of heat treatment of the alloy. The process diagram is shown in Figure 2.

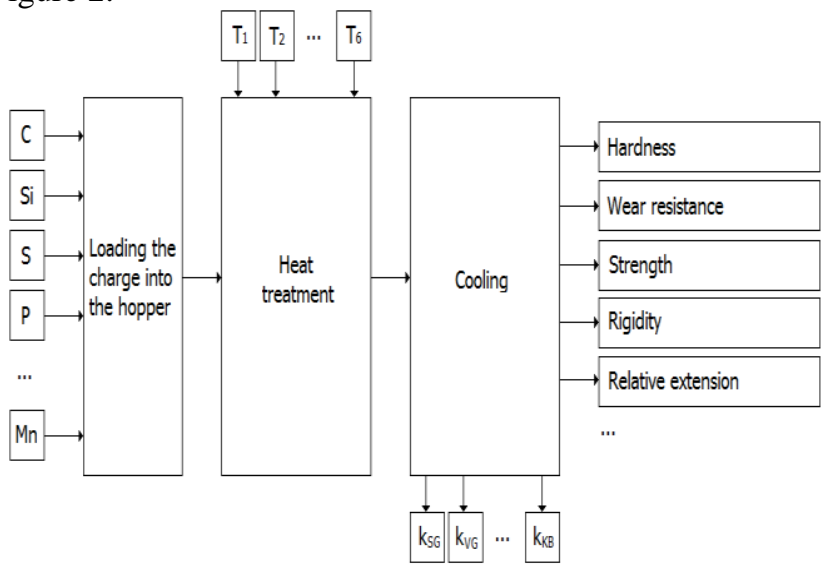

Figure 2. Simplified flow chart

The process is modeled using a probabilistic deterministic finite state machine. The state in which the automaton cells can be located is characterized by a set of numbers in the binary system, depending on the dimension of the output signal containing information about the properties and structure of the alloy. If only two properties are considered priority when optimizing the manufacturing process, strength and rigidity, then in this case the output signal will contain an element $\tau \gamma$ from the set $Y=\{00,01$, $10,11\}$, where 0 means the priority property of the alloy is not fulfilled to the required values, and 1, respectively, execution.

The result of the work of the probabilistic automaton will be the following transition matrix for the probabilistic automaton simulating the process of metal production:

\begin{tabular}{|l|l|l|l|l|l|l|l|l|}
\hline & 000 & 001 & 010 & 011 & 100 & 101 & 110 & 111 \\
\hline 000 & 0 & 0,1 & 0,2 & 0,1 & 0,5 & 0 & 0 & 0,1 \\
\hline 001 & 0 & 0,2 & 0,3 & 0 & 0 & 0,1 & 0,3 & 0,1 \\
\hline 010 & 0,2 & 0,1 & 0,2 & 0,1 & 0,1 & 0,1 & 0 & 0,2 \\
\hline 011 & 0,1 & 0,2 & 0,4 & 0,3 & 0 & 0 & 0 & 0 \\
\hline 100 & 0,2 & 0 & 0 & 0,1 & 0 & 0,3 & 0,1 & 0,3 \\
\hline
\end{tabular}




\begin{tabular}{|l|l|l|l|l|l|l|l|l|}
\hline 101 & 0 & 0 & 0,1 & 0,1 & 0,2 & 0,2 & 0,2 & 0,2 \\
\hline 110 & 0 & 0,1 & 0,2 & 0,3 & 0,2 & 0 & 0,1 & 0,1 \\
\hline 111 & 0,1 & 0,1 & 0,1 & 0,2 & 0,2 & 0,1 & 0,1 & 0,1 \\
\hline
\end{tabular}

\section{CONCLUSION}

The described probabilistic approach based on modeling random factors in the technological process using finite state machines is an essential part of the modern concept of product quality management. The need to identify the causes of the spread of processes, to make decisions based on factual information that contribute to the continuous improvement of the quality of processes and products, determines the use of statistical methods as the formal basis of modern quality management.

This approach will optimize the properties and structure of alloys by varying the chemical composition and parameters of heat treatment of the alloy during production.

\section{REFERENCES}

1. Blyumin S.L., Korneev A.M. Discrete modeling of automation and control systems: Monograph. - Lipetsk: LEGI. - 2005 .- 132 p.

2. Korneev A.M. Modeling complex production systems with using iterative networks / A.M. Korneev, A.M.M. Nagi, T.A. Smetannikova // Fundamental Research. - 2016. No.5-2. - P.260-263.

3. Blumin, S.L. Linear cell machines: the state space approach / S.L. Blumin, R.G. Farajev // Automation and Telemechanics. - 1982. No.2. - P.125-162.

4. Butkovsky, A.G. Structural theory of distributed systems / A.G. Butkovsky. - M .: Nauka, 1977 .- 320 p.

5. Korneev A.M. Optimization of the database structure of multi-stage production systems / A.M. Korneev, L.S. Abdullah, V.A. Lysikov // In the book. New technologies in scientific research, design, management, production: Proceedings of the international scientificpractical conference. - 2017. - P.300-304.

6. Korneev A.M. Evaluation of the effectiveness of the functioning modes of complex systems, taking into account the costs of production / A.M. Korneev, T.A. Smetannikova, A.M. Vasyukov // Bulletin of the Lipetsk State Technical University. - 2015. - No. 2 (24) - P.15-19.

7. Korneev A.M. Search for optimal functioning modes of complex industrial systems / A.M. Korneev, G.M. Al-Sabri // Modern high technology. - 2016. - No. 9-2 - P.233-236.

8. Sakarovitch, Jacques. Elements of automata theory / Jacques Sakarovitch. - Cambridge University Press, 2009. - p. 758.

9. Bukharaev, R.G. Fundamentals of the theory of probabilistic automata / R.G. Bukharaev. - Nauka, 1985 .- 288 p.

10. Korneev A.M. Search strategy for optimal technological modes in discrete cell-hierarchical systems / A.M. Korneev, T.A. Smetannikova, G.M. Al-Sabri, A.M. Nagi // Modern problems of science and education. - 2015. - No. 2-2 - p.150.

11. Korneev, A.M. The use of iterative chains to describe multi-stage spatially distributed production systems / A.M. Korneev, V.N. Kid T.A. Smetannikova // Bulletin of the Peoples' Friendship University of Russia. Series: Engineering Research. - 2012. - No. 2. - P.78-84. 